\title{
Creación de una geodatabase de los archivos geodésicos de la dirección general de catastro y geografía, Honduras
}

Yessica Yamileth Sosa Reyes y Jessica Villatoro

\section{Resumen:}

En el marco de la práctica profesional de la maestría en Ordenamiento y Gestión del Territorio, se desarrolló la creación de una Geodatabase en la Dirección General de Catastro y Geografía (DGCG), la que sería el comienzo para una base de datos más segura y organizada, que permita una mejor distribución de datos geográficos generando para ello una cartografía confiable y altamente calificada.

La creación de la Geodatabase permitió ordenar archivos de la elaboración digital de las hojas cartográficas, mapas departamentales, mapas municipales, mapa oficial del territorio de Honduras y otros que se generan desde el departamento de cartografía de la DGCG.

Para la creación de la Geodatabase se utilizo una metodología la cual consistió en un proceso secuencial de pasos que incluyen: generación de File Geodatabases, Feature data set y Features Class, seguido de la migración de datos digitales en formato shapefile elaborados en el programa Arc View al programa Arc Map de ESRI, en donde se encontraron archivos desde Shapefiles, Tablas, Raster y otros compatibles a la estructura de datos nativa. Al mismo tiempo se realizó una comparación de la base de datos creada con la ya existente, con el fin de detectar posibles deficiencias o mejoras potenciales tanto de la geodatabase como de la manera de creación de los archivos digitales generados dentro de la DGCG.

Se concluye que la creación de una geodatabase para la DGCG permitirá a corto, mediano y largo plazo un manejo adecuado de la información, mejor edición, actualización y administración de datos geográficos del departamento de Cartografía de la DGCG.

Palabras clave: DGCG, Cartografía, Geodatabases(bases de datos), Feature data 
set, Feature Class.

\section{Abstract:}

In the context of professional practice expertise in Planning and Land Management, was developed to create a geodatabase in the Cadastre and Geography (DGCG), which would be the start to a database more secure and organized, which allows a better distribution of geographic data for this mapping generating reliable and highly qualified.

Creating the Geodatabase allowed the development order digital files of map sheets, city maps, city maps, official map of the territory of Honduras and others that are generated from the mapping department DGCG.

For the creation of the Geodatabase methodology was used which consisted of a sequential process steps including: generation File Geodatabases, Feature data set and Features Class, followed by migration of digital data in shapefile format developed in the program Arc View the ESRI ArcMap program, where they found files from shapefiles, tables, and other compatible raster data structure natively. At the same time a comparison of the database created with the existing order to identify any weaknesses or potential improvements of both the geodatabase and the way to create digital files generated within the DGCG.

It is concluded that the creation of a geodatabase for DGCG enable short, medium and long term proper management of information, best editing, updating and management of geographic data department DGCG Mapping.

Keywords: DGCG, Cartography, Geodatabases (satos bases), Feature data set, Feature Class.

Yessica Yamileth Sosa Reyes (yessicasosa@yahoo.com); Jessica Villatoro, (jeki_villatoto@hotmail.com). Universidad Nacional Autónoma de Honduras. Dirección General de Catastro y Geografía. 


\section{INTRODUCCIÓN}

Dentro de las funciones del Departamento de Cartografía de la DGCG, está la de crear, desarrollar y administrar, un sistema nacional de Información territorial en materia de propiedad, creando así; la cartografía, la cual sigue una secuencia de pasos como: el diseño, elaboración y procesamiento, de Mapas Oficiales de Honduras, Hojas Cartográficas, Mapas Departamentales, Mapa Municipales, entre otros. (Instituto de la Propiedad)

Lejos quedan los tiempos en que las hojas cartográficas se elaboraban en formato papel por las instituciones nacionales encargadas de todo el proceso, en donde este era mucho más complejo, es así que en la actualidad los avances en informática y telecomunicaciones, junto al aumento de la capacidad en el manejo de computadoras y programas han transformado radicalmente el modo de producir, tratar e intercambiar cartografía; manejándola de forma digital. (Ruiz López, Coll ALiaga, \& Martínez-llario)

En tal caso el Departamento de Cartografía de DGCG están haciendo logros para transformar la cartografía física a digital, teniendo en cuenta que hasta el momento existe una base de datos digital, que contiene información actualizada de 12 hojas cartográficas, 67 municipios (aproximados) y 14 departamentos; no obstante, la meta es actualizar 281 hojas cartográficas que componen el país.

La base de datos digital del Departamento de Cartografía de la DGCG ha sido diseñada y distribuida en carpetas directamente del disco C:/ y trabajada en el programa Arcview, sin embargo yendo al pie de la vanguardia, hoy en día se está trabajando con el programa ArcGis 10, el cual contiene sus propios iconos y lenguaje para guardar bases de datos ó Geodatabases.

Las geodatabases constituyen un formato de ArcGis de gran utilidad, al poder reunir dentro de un mismo archivo elementos de todo tipo: puntos, líneas, polígonos y elementos raster. El uso de las Geodatabases simplifica la entrega de proyectos a un tercero, dado que si está bien organizada, la información es fácilmente accesible y no hará falta grabar cientos de shapes en carpetas, sino solo geodatabase y el proyecto de archivo tipo *.mxd de ArcGis. (Los SIG y sus aplicaciones prácticas). 
La principal ventaja de manejar la información espacial en una geodatabase y no en archivos del sistema, es que se aprovechan las ventajas del Sistema Gestor de base de Datos Relacional esta incluye: restricciones de acceso y seguridad de la información, soporte para SQL- Structured Query Language- para realizar consultas espaciales complejas, la arquitectura cliente-servidor de la base de datos permite que múltiples usuarios realicen consulta y edición simultánea. (ESRI).

Además las Geodatabases garantizan una alta interoperabilidad, información cartográfica ordenada y jerarquizada, alto control del tipo de dato a introducir así como de su formato (cifras decimales y enteros), no acepta duplicidad, permite restringir los datos utilizando dominios, para así disminuir errores en atributos, permite utilizar y almacenar reglas de topología, e incluso programar reglas propias. (Ciampagna, 2010)

Viendo la importancia de tener datos actualizados, confiables y organizados para generar cartografía calificada por la DGCG, siendo parte del Desarrollo e innovación, se genero una Geodatabase referente al mismo formato que ofrece el programa ArcGis 10 a través de ArcCatalog, con mayores ventajas que una simple carpeta en el disco C:l .

La información que a continuación se presenta es acerca de la Geodatabase creada en la cual la idea fue crear una base de datos actualizada, añadir los archivos shapefile existentes, mediante la importación de archivos.

\section{OBJETIVOS}

\section{General}

Generar una Geodatabase organizada, que permita un mejor manejo, modelado y control de la información geoespacial del departamento de Cartografía de la DGCG.

\section{Específicos}

- Transformar datos cartográficos de formato Arcview a Arcgis del Departamento de Cartografía de la Dirección General de Catastro y Geografía (DGCG).

- Generar, a través de ArcCatalog Geodatabase's, que permitan el despliegue de una carpeta con nombre: ARCHIVO NACIONAL, este a su vez que contenga 
archivos de cada proyecto (Hojas cartográficas), Departamentos y Municipios de Honduras, con sus respectivos shapes.

- Generar un manual de uso de Geodatabase, que permita a los usuarios conocer la técnica de transferir datos de carpetas del explorador de Windows a una geodatabase, para que la misma sea alimentada al crear nuevos proyectos, archivos, tablas, raster u otros elementos necesarios para la elaboración de cualquier trabajo de cartografía digital, necesaria dentro del departamento de cartografía.

\section{METODOLOGIA}

La metodología de trabajo fue desarrollada bajo un diseño de base de datos en donde los archivos correspondientes están conformados por archivos tipo file-Geodataset y Feature Data set representando los Departamentos del territorio nacional de Honduras (divisiones políticas), las Hojas Cartográficas y Municipios.

Teniendo en cuenta cual es el propósito del diseño de una base de datos geo referenciada se enuncia lo siguiente; La base de datos ha sido procesada desde muchos años en forma análoga utilizando procesos donde la elaboración era tardía. En la actualidad existen programas que ayudan a minimizar el tiempo de elaboración de trabajos de cartografía, dentro de ellos; los de la casa comercial ESRI, manejando datos y proyectos digitales en formato Arcview, ahora en su versión actualizada de Arcgis.

Debido a la cantidad de información cartográfica importante para el departamento de Cartografía de la DGCG, se logro diseñar una Geodatabase de manera confortable y amigable, quedando conformada de la manera que se muestra la siguiente figura:

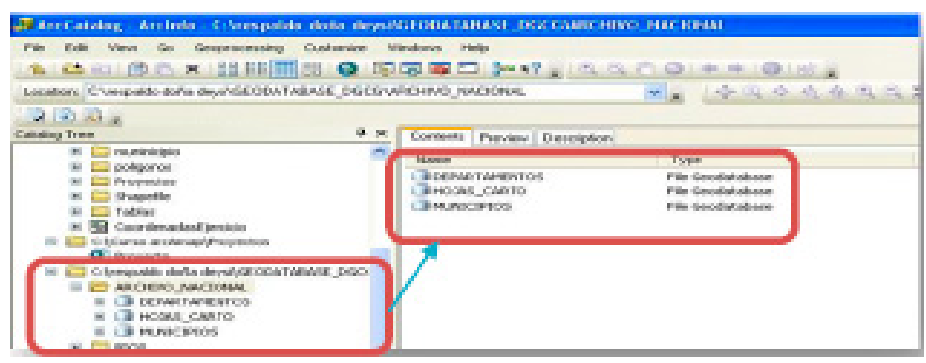

Figura $N^{\circ} 1$ : Archivo Nacional, conteniendo tres Geodatabases 
- DEPARTAMENTOS: Los que comprenden las divisiones político administrativas de cada Departamento de Honduras.

- HOJAS_CARTO: Los que comprende toda las hojas cartográficas del territorio nacional de Honduras.

- MUNICIPIOS: Que comprenden las divisiones administrativas de los municipios de Honduras.

Para cada Geodatabase se realizó la creación de Feature Data Set, así como se muestra en la figura 2:

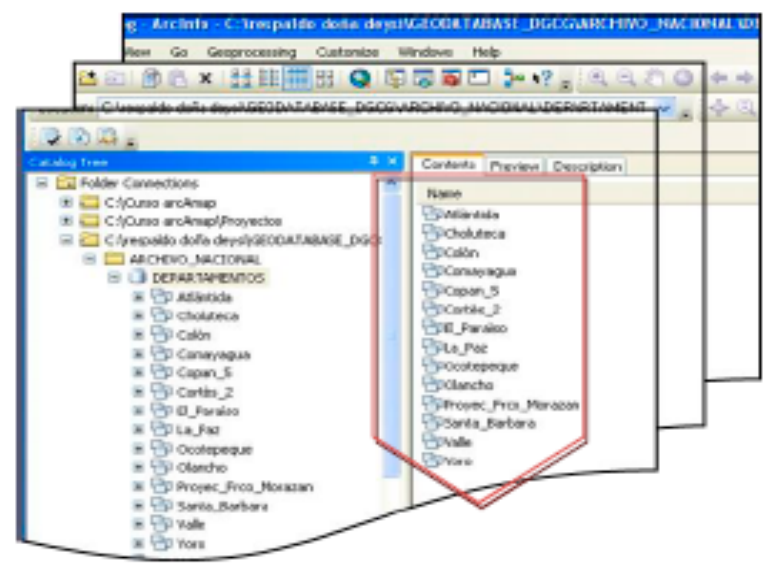

Figura $\mathrm{N}^{\circ} 2$ : Feature data set de 14 Departamentos

Y para cada Feature data set, se genero la creación de Features Class. Los Feature class son los archivos tipo shape que conforman el feature data set, todos aquellos que forman los puntos, líneas y polígonos.

En la siguiente figura se muestran como quedan los archivos tipo shape, pertenecientes a cada Feature Data set. 


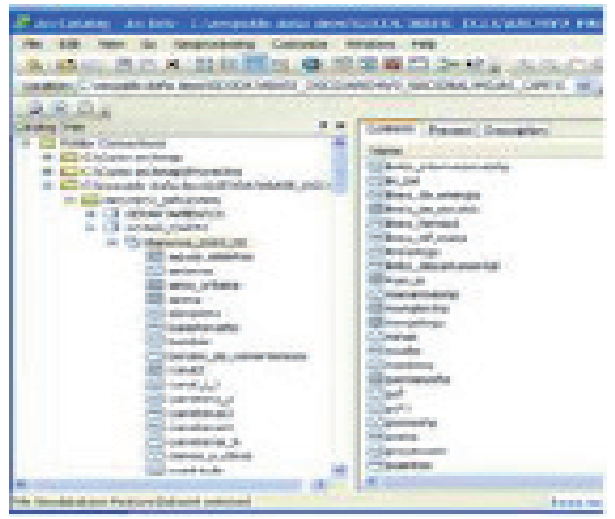

Figura $\mathrm{N}^{\circ}$ 3: shape importados

\section{RESULTADOS}

Dentro de las funciones y actividades que se realizaron están: la transformación de datos en formato arcview a formato arcgis y creación de una geodatabase de la base de datos de la gerencia de cartografía, de la dirección general de catastro y geografía.

La creación de la Geodatabase fue previamente diseñada de manera tal que permita estar en una localización fácil de encontrar dentro del computador en donde se tiene y se maneja la base de datos.

Un aspecto importante a considerar en la elaboración de la Geodatabase es que, al momento de ser creada se definió como su sistema de coordenadas geográficas: WGS 1984 (Word Geodesic System).

Dentro del producto obtenido se tiene un Archivo de Nombre ARCHIVO NACIONAL con tres Geodatabases conformada de los siguientes elementos cartográficos tal y como se muestra en la figura $\mathrm{N}^{\circ} 4$. 


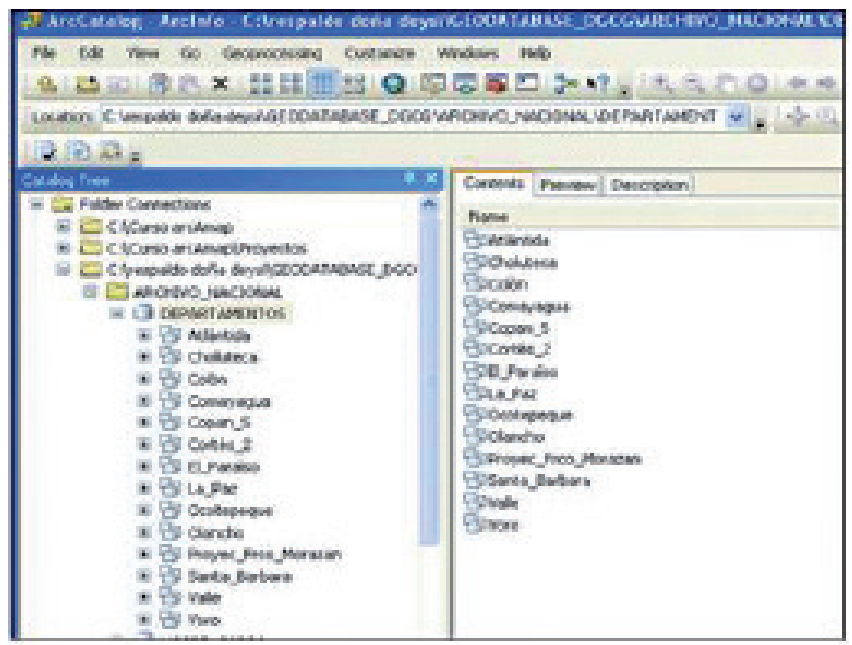

Figura N 4: Diseño de la Geodatabase Propuesta

Además de ello se elaboró un Manual que servirá de base para las elaboraciones, restructuraciones ó rediseños futuros de Geodatabases. Que servirá para alimentación de la misma.

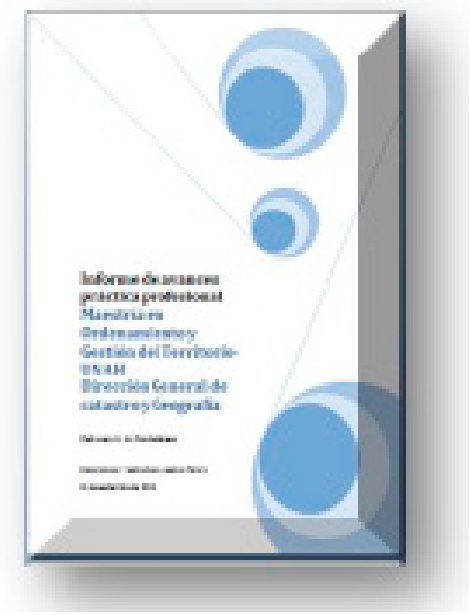

Figura 5. Documento de Informe. 


\section{LOGROS ALCANZADOS Y PRODUCTOS DESARROLLADOS}

Dentro de los logros alcanzados se enumeran los siguientes:

- Una geodatabase identificada con el programa ArcGis.

- Capacitación al personal del Departamento De Cartografía de la DGCG.

- Un manual para la elaboración y alimentación de Geodatabase's.

\section{LIMITANTES}

Dentro de las limitantes se mencionan los siguientes:

- Logística: equipo tecnológico.

- Acceso y organización de la información: no se permite llevar la información del lugar de trabajo y algunos datos están desorganizados encontrándose fuera del computador.

- Tiempo: como practica desarrollada el factor tiempo fue una limitante ya que para una institución del estado encargada de la cartografía hondureña es un trabajo que requiere de mayor atención.

- La geodatabase es una muy buena herramienta de almacenaje de datos pero es limitada, ya que no permite una amplia gama de diseños.

\section{.DISCUSIÓN}

- Es importante que el Departamento de Cartografía de la DGCG maneje la GEODATABASE en ArcGis propuesta, conteniendo información cartográfica relevante, ya que además de acercarse a los cambios tecnológicos y la innovación, es uno de los primeros pasos para organizar la información y gozar de todos los beneficios de la Geodatabase.

- La GEODATABASE actualizada posiciona al Departamento de Cartografía de la DGCG en agentes de cambio, ese cambio permitirá un adecuado manejo de datos y una mejor interacción y trabajo entre los diferentes departamentos de la DGCG, que conlleva a cumplir las metas de dicha Institución Nacional.

- La geodatabase propuesta es capaz de manejarse, importarse o manipularse por otros programas como el Diseño Asistido por Computadoras (CAD), Quantum gis, Map server, entre otros. Esto permite no limitarse a una sola herramienta de trabajo.

- El diseño de la Geodatabase es el mismo que anteriormente maneja la insti- 
tución, el cual incluye un Archivo Nacional que contiene hojas cartográficas, Municipios y Departamentos, pero con otro formato (ArcGis) y de forma reorganizada de tal forma que los shapes se encuentren con el código de Departamento y Municipio, para una mejor búsqueda y evitar la duplicidad de información.

\section{CONCLUSIONES}

- La importancia de una geodatabase dentro de una institución como lo es la Dirección General de Catastro y Geografía, encargada de la cartografía nacional, contribuye grandemente en el ordenamiento territorial, ya que sin cartografía no es posible desarrollar y ejecutar ordenamiento territorial.

- La ventaja de importar la base de datos del Departamento de Cartografía de la DGCG, de Arcview a ArcGis radica en implementar un sistema de posicionamiento, de fácil adaptación y uso, capaz de dar mayor seguridad de datos.

- Logro importarse a la Geodatabase el 19\% de la información, esperando en un futuro tener $100 \%$ de la información importada.

- La elaboración de un manual y el desarrollo de una capacitación sobre la GEODATABASE, al personal del depto. de cartografía de la DGCG, son los inicios de una réplica de conocimientos para la futura organización y alimentación de la GEODATABASE.

\section{BIBLIOGRAFIA}

- Ciampagna, J. M. (12 de Noviembre de 2010). El blog del profe José. Recuperado el 22 de Marzo de 2013, de elprofejose.com/2010/11/12/¿por-que-usar-u na-geodatabase/

- ESRI. (s.f.). Help Arcgis. Recuperado el 04 de Octubre de 2012, de http://help. arcgis.com/es/arcgisdesktop/10.0/pdf/tutorial_building_a_geodatabase.pdf

- Instituto de la Propiedad. (s.f.). Recuperado el 04 de Octubre de 2013, de http:// www.ip.gob.hn/index.php/catastro-y-geografia/acerca-de-catastro

- Los SIG y sus aplicaciones prácticas. (s.f.). Recuperado el 21 de Marzo de 2013, de www.todosig.es/index.html 
- Ruiz Lopez, F., Coll ALiaga, E., \& Martinez-llario, J. (s.f.). Infraestructura de Datos Espaciales de España. Recuperado el 22 de Marzo de 2013, de www. iddee.es/resourses/presentaciones/JIIDE10/ID414_Analisis_y_estructura_de_ una_Geodatabase_BTA.pdf 